\title{
Using Imaging to Introduce Engineering to Freshmen
}

\author{
Paul Laplume, Michael Ruane \\ Electrical \& Computer Engineering, Boston University
}

\begin{abstract}
An independent 7-week module on imaging systems is being offered in spring 2002 to introduce freshmen engineering students to basic methods of problem solving, image analysis tools, and design. It offers several top-down, high-level methodologies for building solutions to interesting challenges, motivating the students to consider engineering as a problem solving discipline, rather than an assemblage of many low-level facts and skills. Preliminary assessment results will be given at the presentation of this paper.
\end{abstract}

The module begins with the presentation of basic digital imaging methods and issues, using PC hardware and web cameras available in the department electronics lab. As student proficiency grows, they advance in teams to the High Tech Tools and Toys Laboratory, which supports five dedicated imaging stations. At each station, the students face a particular imaging problem, which they solve using a variety of hardware and software tools. Solutions are structured to make sure the students can be reasonably successful with their novice level of understanding. The 'Tools and Toys' include different cameras, frame grabbers and digital channels, and imaging software ranging from MATLAB to LabVIEW and Vision Foundry.

Problem solving is emphasized, as is the engineer's choice of appropriate and varied tools. The advanced stations present problems in gauging, pattern recognition, coin counting, tracking, and acoustic imaging. Several also incorporate data interfaces, so that the system can change the environment based on image analysis. The software tools illustrate a range of user control and programming, including menu-driven systems, LabVIEW, MATLAB and C++.

\section{Introduction}

Around 1990 it was suspected that retention of Boston University lower division engineering students was being hindered by the emphasis placed on math and science fundamentals during the first two years, and the low level of freshman student contact with engineering faculty. Math and science courses were taught by non-engineering faculty and presented few opportunities for students to understand and enjoy the fundamental problem solving role of the engineer. Boston University introduced the course 'Introduction to Engineering' to connect freshmen with engineering faculty, to help students explore different engineering majors, and to introduce engineering problem solving early in the curriculum. 


\section{Main Menu}

In 'Introduction to Engineering' a team of faculty typically offers ten different concurrent sevenweek 'modules', twice a semester. Topics are developed in each of the College's four departments to represent each degree program, to reflect faculty interests, and to engage students and encourage their continued study in engineering. Students choose any two of the ten modules to fulfill their semester-long course requirement. Modules meet 13 times, for 2 hours each meeting.

In fall 2000 the National Science Foundation awarded a grant for an Engineering Research Center for Subsurface Sensing and Imaging Systems (CenSSIS) ${ }^{1}$ to Boston University, Northeastern University, Rennselaer Polytechnic Institute, and University of Puerto Rico, Mayaguez. Part of the education plan for CenSSIS includes introducing imaging technology to undergraduates. The plan seeks to generate undergraduate interest in subsurface sensing and imaging, in undergraduate research opportunities related to CenSSIS, and in careers in this area. An initial freshman course was offered at Northeastern in winter 2001, which primarily dealt with acoustic imaging ${ }^{2}$. In 2001 an 'Introduction to Engineering' module on imaging was developed at Boston University through CenSSIS. The module reported in this paper, 'Exploring Hidden Worlds' is being offered for the first time in spring 2002. This paper describes the module and its role in introducing engineering (and CenSSIS) to freshmen.

\section{Exploring Hidden Worlds - Imaging Module Goals}

Four specific goals were set for the module, and presented at the first class:

1. To understand and apply image structures and operations. Students should learn how images are stored and described mathematically, and how basic statistical, ge ometric, and filtering operations are mathematically applied to images.

2. To understand common hardware technologies for collecting images. Students should learn the principles of operation of common image collection hardware, and the standard parameters used to specify that hardware.

3. To understand common software tools for processing images and initiating responses back to the environment. Students should develop basic proficiency with several software products that support image processing. These products should illustrate a range of software complexity and abstraction.

4. To develop basic design skills in imaging and image processing through real-world applications and problem solving. Students should work with engaging problems, using real engineering tools, to see how imaging hardware and software can meet practical needs. They should use or extend the hardware and software to develop their own engineering solution for an open-ended problem.

These are ambitious goals for a seven week module with novice engineering students. Much of the learning has to be 'just-in-time' and problem-oriented. Complete theory cannot be developed without further prerequisite courses and more technical sophistication, but this does not preclude understanding of problem contexts and the development of initial solutions. Faculty experience with earlier modules (on fiber optic communications, radio-controlled cars, and spacecraft instrumentation) showed that considerable learning and problem solving can occur even though

Proceedings of the 2002 American Society for Engineering Education Annual Conference \& Exposition Copyright $\odot$ 2002, American Society for Engineering Education 


\section{Main Menu}

the pace of material can sometimes be overwhelming and the technology may not be completely understood. The focus on introducing engineering to the freshman, rather than building a strong competency, makes this experience and its goals fundamentally different from most of the curriculum. The engaged and interested student will return and take electives or pursue undergraduate lab experiences to learn the fuller story.

\section{The Boston University High Tech Tools and Toys Laboratory (HTTTL)}

CenSSIS support allowed the creation of a small $\left(300 \mathrm{ft}^{2}\right)$ eight-station imaging laboratory called the 'High Tech Tools and Toys Lab' (HTTTL). Five stations were established for the first offering of the freshman imaging module, covering gauging, pattern recognition, counting, dynamic target tracking, and static acoustic imaging. These applications broadly reflect research areas in imaging at Boston University, and are also interesting and intuitively understandable to the freshmen. We will develop additional applications over the next two years.

Class sessions introduce the students to three department labs: electronics, signals, and the HTTTL. The electronics and signals lab have resources to allow each student to work on her own PC. All advanced applications are held in the HTTTL, where students work in teams of two. Each station in HTTTL has a desktop Pentium IV PC, and all experiments are Windows based.

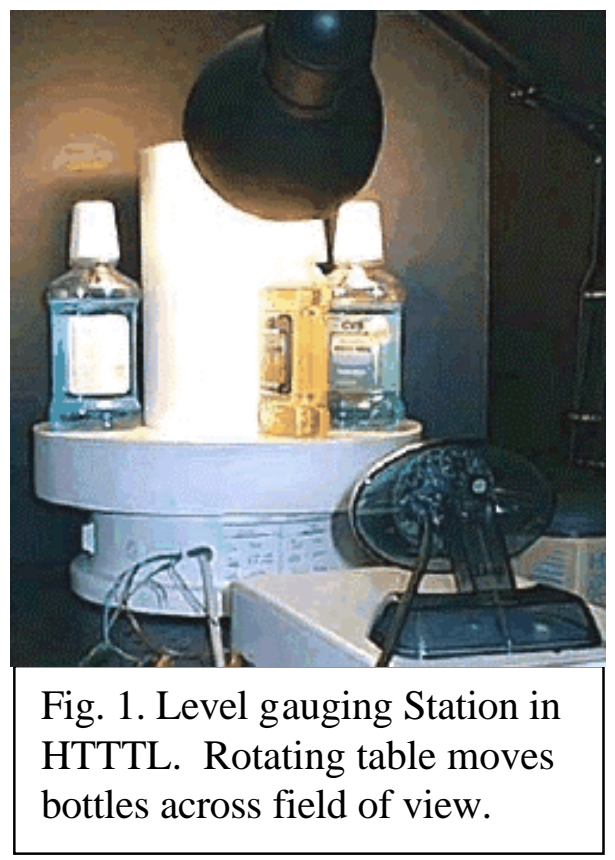

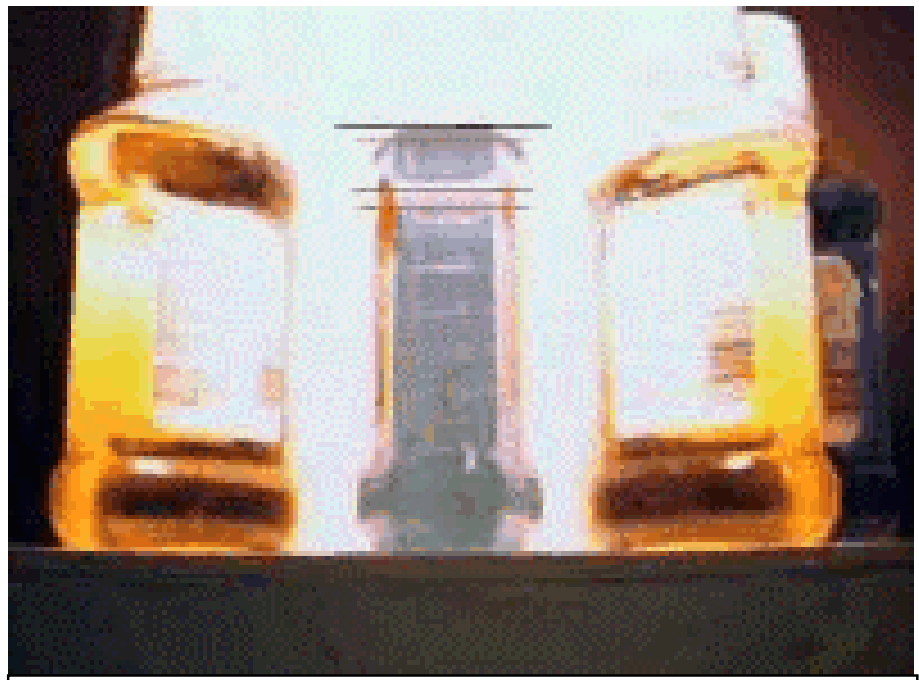

Fig. 2 Image Builder processed frame with horizontal gauge lines on DUT in center of frame.
Each station will now be briefly described, along with its application problem:

\section{Level Gauging Station-Using} FireWire data transfer, a color digital camera records images of mouthwash bottles on a rotating table. Some of the bottles are overor under-filled, and one has incorrect coloring. LabVIEW Image Builder software captures and processes the images to identify out-of-tolerance fill levels. (Fig. 1 and 2). Each student must learn to use the station, individually setting

lighting, rotation speed, and threshold parameters. Each student must then train a second student in the use of the system. After all students have learned the operation of level 


\section{Main Menu}

gauging, they must develop a scheme to adjust the LabVIEW model to identify the bottle with out-of-tolerance coloring. This is submitted as homework, in the form of a structured algorithm for expanding the LabVIEW model. The most interesting homework issues were the definition of the field of view, and the meaning, in LabVIEW, of terms like 'Blue is acceptable', or 'Yellow is out of spec'. They do not actually write new LabVIEW code.

2. Microscopy- Using a Sony camera and image acquisition board, and a Linos microscope, the system images a printed circuit board that has a missing pin and finds the error location. MATLAB, the Image Toolbox, and the Data Acquisition Toolbox control this station. (Fig. 3)

3. Static Pattern Recognition (Coin counting)- A Sony camera, Sony image acquisition board, and a Data Translation digital I/O board, are installed at this station. A script in Vision Foundry image processing software identifies all coins in the field of view, calculates their value, and displays the value using digital I/O board outputs to drive seven-segment displays. Initially quarters, dimes, nickels, and pennies can be recognized; the students must adjust a C++-like script to read halfdollars, and to reject Boston subway tokens. This homework requires the students to understand the reduction of the initial image to blobs with allowable diameters. They have to insert code to accept the larger half-dollar coins, and reject the subway tokens.

4. Dynamic target tracking A FireWire web cam tracks a child's red racecar as it moves around a toy track. Software operations, written in Image Builder, must proceed fast enough to follow the car. Windowing and

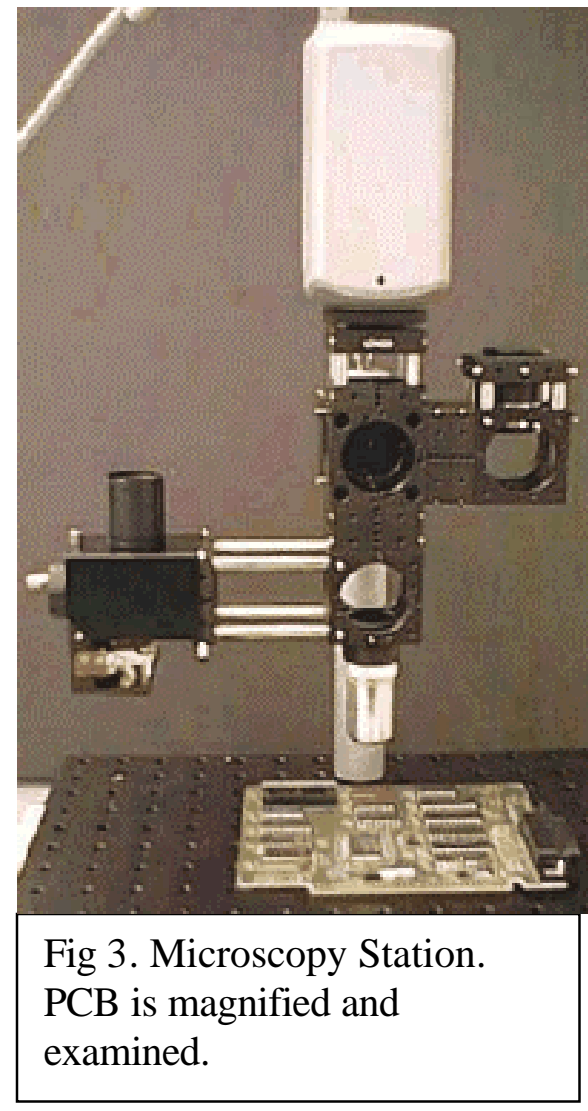
image erosion are used to reduce processing time and image noise. The students see how real-time operations can eventually swamp an application and require performance engineering. The system is closed by feeding the control voltage for the car back to the track, accelerating on the straight track and slowing for curves.

5. Static Acoustic Imaging (originally at Northeastern University) ${ }^{2}$ MATLAB controls a piezoelectric acoustic source/probe to provide acoustic range to a target. Initially the system provides simple line profiles for an acoustically opaque underwater shape. The students must generalize the scanning to two-dimensions and create a solid profile from the range data. Data is collected on a data acquisition card or digital scope.

\section{Module Activities}

Students generally have experience with $\mathrm{C}++$ and some MATLAB, but these are not formal prerequisites. Most have never used a PC for data acquisition or control in a larger system. The 'Exploring Hidden Worlds' module occurs entirely in a laboratory setting. The first two meetings, in the electronics lab, introduce image representation, storage and retrieval, and basic image processing operations. Students then have four meetings in the signals lab, where they run 


\section{Main Menu}

MATLAB under Linux. MATLAB Image Processing Toolbox operations include capture and display, statistical analysis (e.g., gray level distributions), line profiles, image algebra, and simple filtering. Students are encouraged to discover WWW imaging resources ${ }^{3,4}$.

Every third module class has a short in-lab quiz to reinforce basic concepts. Weekly homework allows practice of the main concepts and development of problem solving strategies in anticipation of the HTTTL applications. The first homework focuses on statistics (applied to gray levels); another requires written communications. These are areas freshmen sometimes see as irrelevant to their early engineering studies.

The last seven classes are held in the HTTTL, where students work in teams. Since the stations can be done in any order, the teams work in parallel and move among the stations in subsequent classes. Eventually each team gets to work at each station. Access to the lab is increased by the availability of a graduate assistant and the open scheduling of the HTTTL facility. Enrollments in the first two modules are 8 and 17 students. Evaluation information is being collected this spring and will be reported in the conference session.

\section{HTTTL in the Broader Engineering Curriculum}

Developing a HTTTL need not be an expensive undertaking. Adequate PC stations with a FireWire card and camera, and DAQ card are under $\$ 1500$. Software like MATLAB and LabVIEW can be more expensive than the hardware; we were fortunate to have department lab licenses, so each student could use the software. Senior capstone design teams and undergraduate research students worked on some initial hardware and code for several of the stations. We currently have students working to make the test stations controllable over the Internet, using free LabVIEW web tools, to increase the availability of the systems.

'Introduction to Engineering' (Exploring Hidden Worlds) is only one application of the High Tech Tools and Toys Lab. This facility will eventually support a new elective course in subsurface imaging (planned for spring 2003). Senior capstone design projects in subsurface imaging (e.g. an acoustic imaging system for characterizing different piezo acoustic source configurations) already use HTTTL stations, and this activity is expected to grow. Finally, the HTTTL will be a resource to graduate students needing to try various imaging hardware and software for their research.

We expect to offer the 'Exploring Hidden Worlds' module every spring semester to support growing student interest in CenSSIS. Other department labs are developing new imaging capabilities as subsurface sensing and imaging activities grow through CenSSIS. For example, the signals lab now incorporates basic 2-D filtering on images, and every station has a web cam.

\section{Conclusions}

Keeping freshman interested in engineering while they master lower division math and science fundamentals requires deliberate curriculum efforts. The 'Introduction to Engineering' module 'Exploring Hidden Worlds' challenges freshmen with advanced concepts from imaging and machine vision. The intent is not to create deep understanding of imaging, but to convey the

Proceedings of the 2002 American Society for Engineering Education Annual Conference \& Exposition Copyright $\odot$ 2002, American Society for Engineering Education 


\section{Main Menu}

excitement and creativity involved in solving realistic problems with engineering tools. Students learn basic imaging concepts and the use of common hardware and software for image analysis and understanding. This fundamental knowledge prepares them for a series of advanced applications, created with a deliberate variety of hardware and software. Each application is designed to have a demonstration level challenge, where reasonable effort should lead to success, and a more difficult, open-ended extension, requiring the students (in teams) to develop their own solution. Dedicated imaging equipment supports advanced activities in the High Tech Tools and Toys Lab.

\section{Acknowledgments}

This work was supported in part by CenSSIS, the Center for Subsurface Sensing and Imaging Systems, under the Engineering Research Centers Program of the National Science Foundation (award number EEC-9986821). Discussions with Steve McKnight (Northeastern University), E.C. Everbach (Swarthmore College), Gary Saulnier (Rensselaer Polytechnic Institute), and Rafael Solis-Rodriguez (University of Puerto Rico/Mayaguez) helped shape the opinions in this paper.

\section{Bibliography}

1. http://CenSSIS.neu.edu Home site for all CenSSIS education and research activities. January 2002.

2. McKnight, S.W., Tadmor, G., Cole, W., Everbach, S.W., Ruane, M., 'Teaching Computation to Engineering Freshmen in a "High-Tech Tolls and Toys Lab", proceedings of the 2001 ASEE National Conference, Albuquerque, NM, June 2001.

3. http://www.theimagingsource.com/ General site for imaging news and product information. January 2002.

4. http://www-ismv.ic.ornl.gov/ Large website of imaging technology, research, applications and some image databases. January 2002.

\section{Biography}

MICHAEL RUANE is Associate Professor of Electrical \& Computer Engineering at Boston University. He received the B.E.E. from Villanova University in 1969, his S.M.E.E. from MIT in 1973, and the Ph.D. in Systems Engineering from MIT in 1980. He spent two years as a Peace Corps volunteer in Sierra Leone, was a staff member of the MIT Energy Laboratory from 1973 until 1977 and is a registered professional engineer (electrical). He joined Boston University in 1980 and is a member of the Boston University Photonics Center and of the Center for Subsurface Sensing and Imaging Systems.

PAUL LAPLUME is a graduate student in Electrical \& Computer Engineering at Boston University. He received the B.S.E.E. from Worcester Polytechnic Institute in 2001, and joined Boston University and the Center for Subsurface Sensing and Imaging Systems in September 2001. He is the primary developer of the advanced applications in the HTTTL. 